\title{
O uso do Scratch na Educação Básica: Um relato de experiência vivenciada no PIBID
}

\author{
Anna Raquel da Silva Marinho', Givanaldo Rocha de Souza², Jean Clemisson \\ Santos Rosa ${ }^{3}$, Pauleany Simões de Morais ${ }^{1}$ \\ ${ }^{1}$ Instituto Federal de Educação, Ciência e Tecnologia do Rio Grande do Norte (IFRN), \\ Campus Natal - Zona Norte - Brasil \\ ${ }^{2}$ Instituto Federal de Educação, Ciência e Tecnologia do Rio Grande do Norte (IFRN), \\ Campus Parnamirim - Brasil \\ ${ }^{3}$ Departamento de Ciência da Computação - Instituto de Matemática. Universidade \\ Federal da Bahia (UFBA) - Salvador - BA - Brasil \\ anna.raqueldescolar.ifrn.edu.br, givanaldo.rocha@ifrn.edu.br, \\ jean.rosa@ufba.br, pauleany.morais@ifrn.edu.br
}

\begin{abstract}
This paper presents an analysis of pedagogical contributions of Scratch, an educational software based on a block language, in basic education. Related experiences are from Institutional Scholarship Program Introduction to Teaching (PIBID) actions at public schools through literature review from authors whose works concern on the object of study in question. This study makes possible an analysis of experiences lived in basic education, as well as the report of actions developed through a workshop, carried out by students of the Degree Course in Informatics from Federal Institute of Education, Science and Technology of Rio Grande do Norte, Campus Natal - North, PIBID fellows.

Resumo. Este artigo tem o objetivo de apresentar uma análise sobre as contribuições pedagógicas do Scratch, um software educacional baseado em uma linguagem de blocos, particularmente na educação básica. São relatadas as experiências vivenciadas no Programa de Bolsas de Iniciação à Docência (PIBID), mediante revisão de literatura de autores cujos trabalhos focam no objeto de estudo em questão. Esse estudo possibilita uma análise de experiências vivenciadas na educação básica, bem como o relato das ações desenvolvidas por meio de uma oficina, realizada por estudantes do curso de Licenciatura em Informática do Instituto Federal de Educação, Ciência e Tecnologia do Rio Grande do Norte, Campus Natal - Zona Norte, que são também bolsistas do PIBID.
\end{abstract}

\section{Introdução}

No século em que vivemos, a computação tem se apresentado como uma ferramenta de fundamental importância na sociedade, seja nos meios de comunicação, industrial, automobilístico, bem como em processos educacionais. Dessa forma, não podemos restringir o ensino da computação apenas em como usar o computador e seus sistemas operacionais e softwares, mas podemos usufruir de diversos recursos que auxiliam no 
processo de ensino-aprendizagem, inclusive nas possibilidades de resolução de problemas do cotidiano. Essas características resultam em novas dinâmicas para o contexto educacional no favorecimento de possibilidades para o aprendizado, tendo em vista que a nova lógica de ensino objetiva não adaptar o modelo de ensino aos equipamentos tecnológicos, mas utilizar os sistemas de informação e comunicação em seu favor.

Segundo Blinkstein (2008), é necessário "saber usar o computador como um instrumento de aumento cognitivo e operacional humano". Assim, após estudos sobre softwares educacionais e as suas contribuições para a educação, com base na estratégia de ensino solução de problemas, selecionamos o software educacional Scratch para ser utilizado na Oficina de Iniciação à Programação. Outros softwares analisados foram App Inventor, Alice e Kodu. Um estudo apresentado por Bombasar et al. (2015) sobre o ensino de computação evidencia o crescente uso desses softwares no desenvolvimento do pensamento computacional diante de uma revisão de literatura nos diversos meios científicos entre os anos de 2006 a 2015. As contribuições pedagógicas do Scratch são relatadas mais adiante no artigo.

O presente artigo tem o objetivo de apresentar uma análise sobre as contribuições pedagógicas do software educacional Scratch, particularmente na educação básica, em experiências vivenciadas no PIBID. A pesquisa relatada foi realizada por meio de revisão da literatura a partir de autores que têm seus trabalhos com o foco no objeto de estudo em questão, cujas referências são a Revista Brasileira de Informática na Educação (RBIE) e congressos cuja temática envolve Informática e Educação, tais como o Congresso da Sociedade Brasileira de Computação (CSBC) e o Congresso Brasileiro de Informática na Educação (CBIE).

Dentre os autores e artigos analisados, destacam-se Pereira e Rapkiewicz (2004), Silva et al. (2008), Gomes e Melo (2013), França et al. (2014) e Ramos (2014), permitindo uma análise a partir das experiências vivenciadas pelos respectivos autores na educação básica de instituições públicas, bem como o relato de experiência das ações desenvolvidas, por meio de uma oficina, realizada por estudantes do curso de Licenciatura em Informática do Instituto Federal de Educação, Ciência e Tecnologia do Rio Grande do Norte (IFRN), Campus Natal - Zona Norte, bolsistas do Programa Institucional de Bolsa de Iniciação à Docência (PIBID), junto a um grupo de alunos do Ensino Médio da Escola Estadual Professora Ana Júlia de Carvalho Mousinho, localizada na Zona Norte de Natal/RN.

Inovar é preciso, e assim como ocorre em todas as áreas, a educação não pode ser deixada de lado. Os recursos são diversos e os professores dispõem de estratégias que podem ser utilizadas no intuito de tornar as aulas mais atrativas e envolver o aluno no processo de ensino-aprendizagem. Portanto, sabe-se que "é importante que juntamente com a inserção do computador na vida dos alunos, o método de ensinar e o conteúdo ensinado sofram alterações que permitam o uso efetivo e qualitativo dessa ferramenta tecnológica" [SILVA; ROMANI; BARANAUSKAS 2008, p. 31]. Já não se faz necessário permanecer com a mesma metodologia de décadas atrás, até porque os recursos são mais acessíveis.

Assim, este artigo apresenta em suas seções algumas considerações sobre as perspectivas para o ensino de computação, por meio da utilização do software educacional 
VI Congresso Brasileiro de Informática na Educação (CBIE 2017)

Anais do XXIII Workshop de Informática na Escola (WIE 2017)

Scratch. Na Seção 2 serão relatados alguns problemas advindos da educação tradicional que acarretam dificuldades na operacionalização na educação básica. Na Seção 3, as possibilidades pedagógicas nas quais o Scratch possa vir a contribuir de forma significativa. Na Seção 4 serão abordadas algumas contribuições que a utilização do Scratch pode promover na formação dos alunos da educação básica. Por fim, na Seção 5, as considerações finais acerca das experiências vivenciadas.

\section{Dificuldades no ensino de programação na educação básica}

A escassez de informações e conhecimentos sobre a área tecnológica ainda se faz presente entre os jovens e nas escolas públicas de ensino básico no Brasil. No entanto,

as soluções computacionais estão presentes em problemas de diversas áreas e atividades humanas, promovendo oportunidades de emprego e inserção de jovens em um mercado globalizado e de alta competitividade. De um lado, apesar dessa demanda, muitos jovens deixam de cogitar uma carreira na área devido à falta de conhecimento dos princípios fundamentados pela Ciência da Computação, construindo uma ideia equivocada da mesma. Por outro lado, parte desses jovens ignora o fato de que boa parte das atividades de computação não é restrita apenas à área de computação [FRANÇA et. al. 2014, p. 1475].

Outro fator complicador para a aprendizagem é "o desenvolvimento do raciocínio lógico em contraste com a prática de memorizar o conteúdo" [GOMES e MELO 2013, p. 652], como consequência da velha metodologia, na qual o professor transmite conhecimento e o aluno decora. Sendo assim, "a falta de motivação do aluno gerada pelo despreparo ou mesmo desânimo quando o mesmo encara a disciplina como um obstáculo difícil de ser superado" [GOMES e MELO 2013, p. 652]. Outros pontos críticos são a dificuldade na interpretação do problema, antes mesmo da dificuldade de interpretação de algum tipo de representação, e a dificuldade na aplicação das suas habilidades prévias, gerando medo e frustração [PEREIRA 2004; RAPKIEWICZ et al. 2006].

Tais dificuldades precisam ser amenizadas ao longo do processo de ensinoaprendizagem, até que sejam completamente superadas. Para isso, existem alternativas como a Computação Desplugada, ou seja, sem fazer uso do computador, bem como com a utilização do computador por meio de softwares educacionais com o intuito de compreender e desenvolver o pensamento computacional na resolução de problemas do cotidiano. Essa última é a alternativa na qual este artigo se baseia.

\section{Contribuições pedagógicas do Scratch}

De distribuição gratuita, o Scratch é um projeto do grupo Lifelong Kindergarten desenvolvido pelo Media Lab, grupo de pesquisa do Massachusetts Institute of Technology (MIT). Este software ajuda os jovens a aprender a pensar de maneira criativa, refletir de maneira sistemática e trabalhar de forma colaborativa habilidades essenciais para a vida no século XXI. Desenvolvido para que o usuário possa programar suas próprias histórias interativas, jogos e animações e compartilhar suas criações com outros membros da comunidade online. Foi projetado especialmente para estudantes entre $8 \mathrm{e}$ 16 anos, mas é usado por pessoas de todas as idades. O Scratch é usado em mais de 150 
países e está disponível em mais de 40 idiomas [SCRATCH 2015]. O processo de ensinoaprendizagem ocorre por meio de uma linguagem visual que permite a manipulação de mídias, tais como imagens e músicas, para a criação de histórias interativas, de jogos ou de animações [MALONEY et al. 2010; MALONEY et al. 2008].

Acreditando na facilidade de programação fornecida pelo Scratch, Malan e Leitner (2007) obtiveram resultados positivos em sua utilização como ferramenta de apoio ao ensino da disciplina introdutória de programação na Universidade de Harvard. Neste aspecto, o uso de uma linguagem de programação visual é especialmente importante, porque os estudantes não necessitaram apropriar-se da sintaxe de uma linguagem de programação tradicional [GOMES e MELO 2013]. Os estudantes demonstraram motivação, engajamento e interesse pelos conteúdos explanados, bem como uma apropriação significativa dos conceitos. O ambiente colaborativo, permeado por desafios, estimula os estudantes a aprenderem a trabalhar em equipe e resolver de modo criativo e autônomo os problemas propostos. Neste ambiente, os estudantes podem compreender os conceitos básicos de programação, na medida em que desenvolvem aplicativos [GOMES e MELO 2013].

A autonomia é uma das orientações didáticas sugeridas nos Parâmetros Curriculares Nacionais (PCNs) no que se refere à capacidade de posicionar-se, ter discernimento, poder de decisão, organizar-se em função de metas, estabelecer critérios, planejar a realização de uma tarefa, identificar formas de resolver um problema, formular boas perguntas e boas respostas, levantar hipóteses e buscar meios de verificá-las, validar raciocínios, resolver conflitos [RAMOS 2014].

A estratégia com solução de problemas é bastante utilizada no ensino da matemática, mas não se restringe somente a essa área do conhecimento. Pode ser considerada como um processo de construção do conhecimento, tendo em vista que pode apresentar o estímulo, ampliação e a percepção de acontecimentos cotidianos e profissionais [ANASTASIOU 2005]. Essa estratégia também possibilita ao estudante desenvolver diversas habilidades como, por exemplo, a criatividade, o raciocínio lógico, reflexão, criticidade, entre outras, para a resolução de problemas. Inclusive a autonomia de utilizar diversos caminhos diferentes que o levem a chegar à resposta mais correta $\mathrm{e}$ que soluciona o problema.

\section{Relato de experiência da utilização do Scratch nas ações do PIBID}

A abstração dos conhecimentos depende, em sua essência, da forma em que eles são apresentados. Por consequência, não é viável simplesmente descaracterizar a importância da aprendizagem escolar em detrimento dos recursos tecnológicos informacionais. Mesmo que exista a informação e que haja um processo cognitivo aliado, se faz necessário a orientação que está presente no contexto escolar [LIBÂNEO 2000]. Cabe aos educadores orientar os seus alunos sobre como utilizar a tecnologia de forma proveitosa para a produção do conhecimento. Nesse caso, o licenciado em Informática tem um papel essencial na sistematização de conhecimentos relacionados ao desenvolvimento do pensamento computacional.

As ações realizadas na Escola Estadual Professora Ana Júlia de Carvalho Mousinho, localizada na Zona Norte de Natal/RN, aconteceram por meio da Oficina do 
Subprojeto Informática do PIBID, planejada e ministrada por duas bolsistas, licenciandas em Informática. Tendo como proposta o Ensino de Iniciação à Programação com o Software Educacional Scratch, cujo intuito foi desmistificar o conceito popular de que o professor de Informática apenas ministra aulas de informática básica, podendo também atuar no desenvolvimento de habilidades voltadas à resolução de problemas, proporcionando o estímulo do raciocínio lógico, estratégias e autonomia.

Assim, foram planejadas aulas teóricas e práticas (duas aulas por semana, com carga horária de 1h cada) para a Oficina de Informática, trabalhando os conceitos dos elementos que compõem o Scratch, visto que neste software "a programação dispensa a digitação de código e se baseia em arrastar e soltar blocos de comandos" [BINI e KOSCIANSKI 2009, p. 6]. Haviam também desafios dos quais, caso os alunos não conseguissem resolver sozinhos, as dúvidas deveriam ser expostas para que resolvessem em conjunto. Participaram da oficina 16 alunos (cinco cursando o $1^{\circ}$ ano, oito cursando o $2^{\circ}$ ano e três cursando o $3^{\circ}$ ano) do Ensino Médio, com idade entre 15 e 20 anos, todos do turno matutino.

A execução da oficina aconteceu de acordo com os seguintes tópicos: 1. Introdução à lógica de programação; 1.1. O que é algoritmo? 1.2. A hora do código: Hour of Code (code.learn.org); 2. O que é o Scratch?; 2.1. Conhecendo a plataforma; 3. Blocos de comandos; 3.1. Estruturas Condicionais: se.. então; 3.2. Estruturas de Repetição: Enquanto, repita.. até; 4. Funções e utilidades do Software; 5. Criação de um projeto.

\subsection{Descrição das aulas}

Após apresentar o conteúdo do módulo, iniciamos o primeiro tópico: Introdução à lógica de programação, construindo o conceito junto aos alunos do que é o algoritmo, em seguida foi pedido aos mesmos que acessassem o site http://code.org, no qual eles deveriam participar da Hora do Código, que possui cursos de introdução à lógica de programação, criado com o intuito de mostrar que se pode aprender o básico da programação de maneira lúdica, independente da faixa etária.

Na segunda aula foi dada continuidade à Hora do Código, dessa vez com outro desafio. Todos conseguiram finalizar ambos os desafios e receber o seu certificado, quanto ao desempenho no decorrer dos níveis, alguns conseguiram concluir mais rápido e outros um pouco mais devagar. Na terceira aula iniciamos o segundo tópico proposto (O que é o Scratch?). Antes de apresentarmos os conceitos, retomamos alguns elementos que já haviam sido expostos no início da oficina. Construímos junto aos alunos a definição do Scratch, demonstramos o layout da plataforma e os principais componentes que o constituem (sprites, palco, cenários, blocos de comandos). Após a apresentação, solicitamos que acessassem o site do Scratch (http://scratch.mit.edu) para que explorassem a plataforma e os componentes apresentados.

$\mathrm{Na}$ quarta aula, a primeira aula da criação de um projeto no Scratch, começamos a introdução do primeiro tópico dos blocos de comandos, o bloco Movimento e seus componentes. Explicamos cada componente deste bloco e pedimos para que os alunos testassem cada um deles, na plataforma virtual do Scratch, observando o seu funcionamento. 
Na quinta aula apresentamos o segundo tópico dos blocos de comandos, o bloco Aparência e seus componentes. Após a explicação, solicitamos que os alunos criassem livremente um programa utilizando o bloco de comandos apresentado na aula anterior e nesta aula. Todos conseguiram fazer as atividades usando os dois blocos.

A sexta aula foi reservada para que os alunos criassem programas no Scratch, seguindo as orientações de uma lista contendo 10 questões, utilizando os blocos de comandos apresentados nas aulas anteriores. Na sétima aula corrigimos a lista. Nesta oportunidade notamos que alguns alunos tiveram dificuldades em implementar os programas. Assim, foi preciso mostrar passo a passo no Scratch os blocos que permitiam os programas funcionarem corretamente de acordo com as orientações da lista.

$\mathrm{Na}$ oitava aula trabalhamos com dois tópicos dos blocos de comandos: Variáveis e Eventos. O motivo de termos explicado os componentes desses dois blocos na mesma aula foi devido os alunos estarem com dificuldades em executar os programas criados, sendo o bloco Eventos primordial para essa ação. Para explicar o bloco Variáveis utilizamos exemplos ligados ao dia a dia dos alunos. Após explicar a função de cada componente, sugerimos que os alunos criassem programas contendo os quatro blocos de comando que foram apresentados.

Na nona aula explicamos o quinto tópico dos blocos de comandos: Sensores. Após a explicação solicitamos que resolvessem duas questões que contemplavam os cinco blocos vistos até o momento. Percebendo a dificuldade de alguns alunos em utilizar o Scratch, resolvemos fazer as seguintes perguntas: O que você está achando do software Scratch? Qual a maior dificuldade que está tendo para resolver as questões propostas? Os alunos deveriam enviar as respostas por e-mail.

Identificamos que todos os alunos estavam gostando do software, o caracterizaram como "fácil", "legal" ou "interessante". No entanto, ressaltaram que sentiam dificuldade de encontrar os componentes adequados para solucionarem os problemas propostos. Desse modo, alteramos a estratégia de aplicação das listas, demonstrando a resolução de problemas semelhantes, para em seguida entregarmos as listas. Notamos que dessa forma os alunos tiveram um desempenho mais significativo. Essa primeira etapa da Oficina foi realizada durante 15 aulas ( $1 \mathrm{~h}$ por aula).

Para trabalhar a autonomia, a criatividade e o trabalho em grupo, a nossa proposta para a segunda etapa da oficina foi a formação de grupos para a produção de um projeto. Cada grupo precisava escolher um tema com fins educacionais. No entanto, os grupos eram responsáveis por escolher como os projetos seriam desenvolvidos no Scratch, por meio da criação de jogos, histórias ou mesmo animações.

Foi solicitado que cada grupo justificasse a escolha do tema do seu projeto. Foram formados cinco grupos (quatro grupos com três integrantes e um grupo com quatro integrantes). Os nomes escolhidos para os projetos foram Pong, Health Life, Não ao preconceito, Sheldon e Calculadora Infantil. O desenvolvimento do projeto teve duração de dois meses (outubro e novembro de 2015, respectivamente).

\subsection{Descrição dos projetos desenvolvidos pelos alunos}

O projeto do Grupo 1, ilustrado na Figura 1, consistiu em um jogo com dois objetos 
VI Congresso Brasileiro de Informática na Educação (CBIE 2017)

Anais do XXIII Workshop de Informática na Escola (WIE 2017)

(bolas), um cronômetro marcando 60 segundos, dois placares mostrando a pontuação de cada objeto e duas barras laterais (uma na lateral esquerda e outra na lateral direita). A função das barras laterais é impedir que os objetos toquem na faixa vermelha.
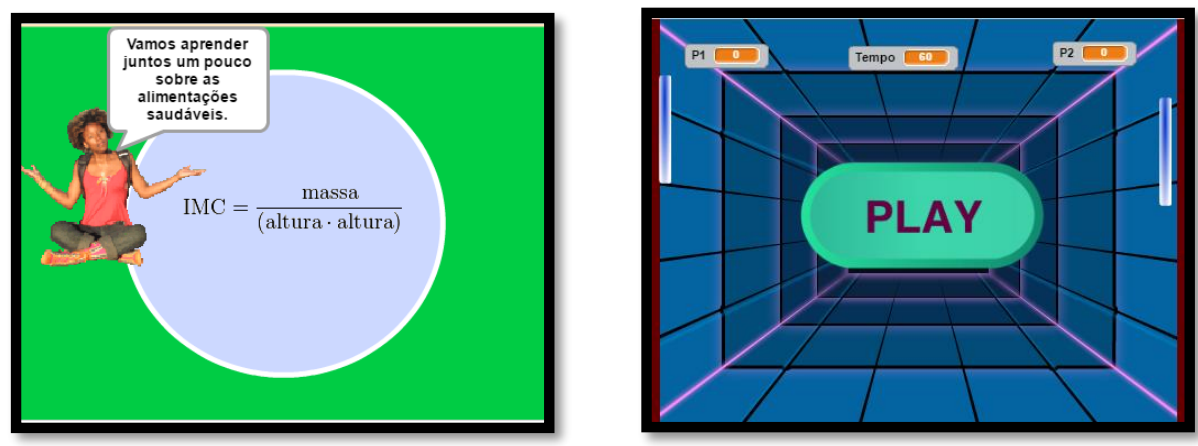

Figura 1. Projeto Pong (à esquerda) e Projeto Health Life (à direita)

O Grupo 2 criou o jogo Health Life, ilustrado na Figura 1, se preocupando com a orientação para uma alimentação saudável, justificando que a função do jogo é que as crianças descubram que devem se alimentar com alimentos saudáveis que ajude no seu crescimento. O projeto, em forma de quiz, mostra alguns tipos de alimentos e pede que o usuário informe qual o alimento que possui a substância " $x$ ", se o usuário acertar recebe uma resposta positiva e informações nutricionais sobre o alimento. Se o usuário errar recebe uma resposta negativa e em seguida as informações nutricionais. Ao final do quiz tem-se a opção de calcular o Índice de Massa Corporal (IMC), recebendo uma avaliação do resultado, se está acima, abaixo ou no peso indicado para a altura.

O Grupo 3, com o projeto Não ao Preconceito, ilustrado na Figura 2, teve a preocupação em trabalhar com um assunto que é bastante discutido na sociedade: o preconceito racial. Justificando que ainda existe muito preconceito na nossa sociedade, por isso o projeto em forma de história acontece em um corredor escolar. A função é conscientizar as pessoas de que todos somos iguais. A história acontece por meio do diálogo entre o diretor e alunos de uma escola que demonstram preconceito ao receber um aluno negro na turma. Os cenários mudam no decorrer do diálogo.
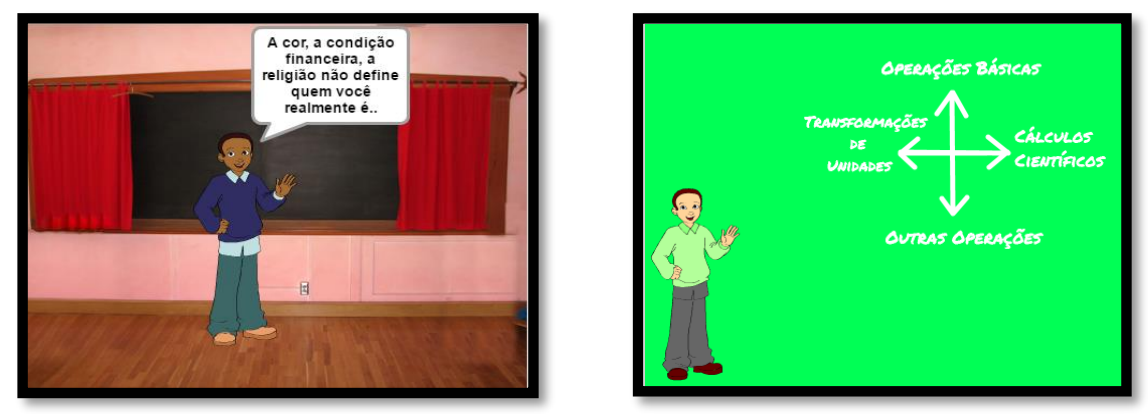

Figura 2. Projeto Não ao Preconceito (à esquerda) e Projeto Sheldon (à direita)

O Projeto Sheldon do Grupo 4, ilustrado na Figura 2, é um projeto lúdico, cuja justificativa do grupo foi que o intuito do programa é auxiliar quem tem dificuldades em operações da física como transformação de unidades, os cálculos da velocidade e a aceleração média, além das funções básicas da matemática. O projeto apresenta opções 
de cálculos, conforme a escolha do usuário, faz-se a explicação da equação utilizada para a realização do cálculo e em seguida é solicitado ao usuário os valores que ele deseja calcular. Esse projeto funciona como uma calculadora com função explicativa.

O projeto Calculadora Infantil do Grupo 5, ilustrado na Figura 3, teve como justificativa incentivar as crianças a aprender matemática com o Scratch de uma forma diferente e divertida, de forma que eles compreendam o que estão fazendo e com figuras que são influência nesse momento da vida. O projeto em forma de calculadora lúdica, contendo as quatro operações básicas, propicia às crianças calcular brincando.

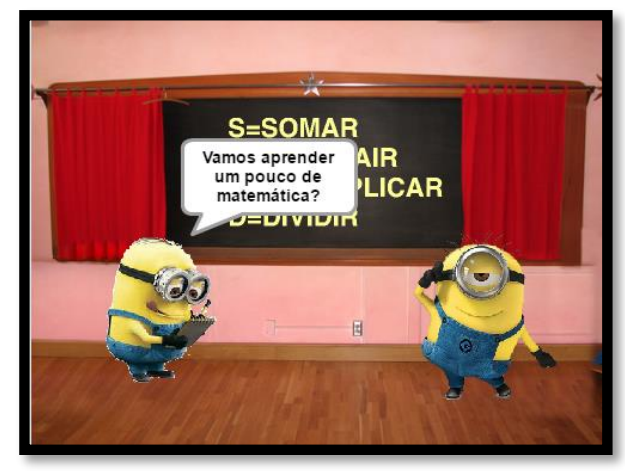

Figura 3. Projeto Calculadora Infantil

No final de novembro de 2015, todos os projetos foram apresentados e testados na Oficina de Informática. Nas apresentações, os grupos justificaram para a turma o motivo da escolha do tema, falaram sobre as dificuldades que surgiram durante a implementação, executaram os projetos e em seguida mostraram os blocos utilizados. Posteriormente, os projetos foram apresentados na Feira de Tecnologia, Arte e Cultura da escola, onde alunos, professores, pais e a comunidade puderam conhecer e manipular os projetos desenvolvidos.

\subsection{Lições aprendidas}

Com essa experiência, pudemos observar que todos os grupos tiveram a percepção das possibilidades de criação que o Scratch apresenta, bem como a preocupação na criação de projetos que possibilitem o aprendizado do aluno, tendo em vista as dificuldades encontradas nas disciplinas escolares que já cursaram ou estão cursando. Essa percepção é destacada na fala de um dos grupos: "o público alvo do nosso projeto seriam alunos do ensino médio, principalmente aqueles que estão iniciando agora o curso, já que nos incluímos nisso e conhecemos bem essas dificuldades" (Grupo 4).

Ao final da oficina aplicamos um questionário em que uma das perguntas foi " $\mathrm{O}$ que você mais gostou na Oficina?". Destacamos as respostas de dois alunos que representam os depoimentos dos demais: "[...] a facilidade do programa onde aprendemos a criar jogos que podem nos auxiliar" (Aluno 1) e "o scratch, que me ajudou a descobrir vários métodos legais de aprender matemática, física, química e outras coisas legais" (Aluno 2). Evidenciamos ainda que a utilização de conteúdos que fazem parte de disciplinas como física, biologia, matemática e história, remetem à possibilidade de trabalhar interdisciplinarmente a informática com as demais áreas do conhecimento. 
As ações desenvolvidas na escola pública partem do pressuposto da necessidade propiciar atividades de desenvolvimento do pensamento computacional. França et al. (2013, p. 282) esclarecem que as ações servem para:

[...] começar a desenvolvê-las e divulgá-las e, principalmente, discuti-las pode ser o caminho para consolidação de práticas educacionais mais condizentes com a nova realidade que a revolução tecnológica e cientifica tem propiciado.

Nessa perspectiva, entende-se que os objetivos foram alcançados, pois o estudo e o desenvolvimento de ações com Scratch possibilitam uma inclusão do aluno da escola pública em vivências de práticas didático-pedagógicas com caráter interdisciplinar, bem como a inserção em uma realidade tecnológica. Além disso, propicia aos licenciandos do PIBID realizarem atividades que contribuam para a constituição dos saberes docentes com fundamentos teóricos e de metodologias de ensino voltadas ao ensino de computação nas escolas públicas. Sabe-se que tal ensino encontra-se em experiências iniciais, no entanto, os resultados alcançados mostram, inclusive na literatura, a relevância as ações de formação nas escolas.

\section{Considerações finais}

Inicialmente, não se imaginou a dimensão que as ações desenvolvidas na oficina teriam, contudo, a participação dos alunos na Feira de Tecnologia, Arte e Cultura da escola, no início de dezembro de 2015, divulgando os seus projetos, projetos idealizados por eles, concretizaram a percepção de que o Scratch contribui significativamente para a formação educacional, tendo em vista que se tentou desenvolver uma perspectiva interdisciplinar perante o projeto em execução na Escola, uma vez que os projetos desenvolvidos pelos alunos abordaram temáticas relacionadas às disciplinas de Física, Biologia, Matemática e História.

A avaliação feita por parte dos alunos, por meio de questionário aplicado ao final da Oficina, mostrou que os objetivos da proposta foram alcançados, por mais que tenha havido algumas dificuldades na resolução dos desafios que foram propostos durante o percurso. Observa-se que a experiência de inclusão do ensino de programação na escola pública possui significativa relevância. No entanto, ainda vivenciam-se problemáticas para o desenvolvimento de formações que contribuam para essa inclusão ser efetivada.

Algumas das problemáticas para que as ações sejam desenvolvidas são profissionais da área qualificados, equipamentos adequados, disponibilidade dos professores das diversas áreas para participar de cursos de formação de iniciação à programação, dentre outras. Assim, as ações que envolvem a iniciação à programação não necessitam apenas serem idealizadas no campo do currículo e sim em uma mediação técnica e humana que efetivamente viabilizem o seu desenvolvimento.

A experiência com a oficina mostrou que, mesmo com a utilização superficial de softwares de iniciação à programação nas escolas de educação básica, é possível a operacionalização de um trabalho didático pedagógico significativo. Além disso, tem-se a possibilidade de vivenciar práticas de formação inicial de professores por meio de experiências com ensino de programação na Educação Básica. 
VI Congresso Brasileiro de Informática na Educação (CBIE 2017)

Anais do XXIII Workshop de Informática na Escola (WIE 2017)

\section{Referências}

Bini, E. M. e Koscianski, A. (2009) “O ensino de programação de computadores em um ambiente criativo e motivador", Encontro Nacional de Pesquisa em Educação em Ciências, Florianópolis.

Blinkstein, P. (2008) "O pensamento computacional e a reinvenção do computador na educação", Disponível em http://www.blikstein.com/paulo/documents/online/ol_ pensamento_computacional.html, Acesso em jan. 2015.

Bombasar, J. R., Santiago, R., Miranda, E. M. e Raabe, A. L. A. (2015) "Ferramentas para o ensino-aprendizagem do pensamento computacional: onde está Alan Turing?", IV Congresso Brasileiro de Informática na Educação, X Conferência LatinoAmericana de Objetos e Tecnologias de Aprendizagem (CBIE-LACLO), Anais do XXI Workshop de Informática na Escola, Maceió.

França, R. S. et al. (2013) "Despertando o interesse pela Ciência da Computação: Práticas na Educação Básica”, VIII International Conference on Engineering and Computer Education, Luanda.

França, R. S. et al. (2014) "Disseminação do pensamento computacional na educação básica: lições aprendidas com experiências de licenciandos em computação", Anais do XXXIV Congresso da Sociedade Brasileira de Computação, p. 1473-1482, Brasília.

Gomes T. C. S e Melo, J. C. B. (2015) “O Pensamento Computacional no Ensino Médio: Uma Abordagem Blended Learning”, Disponível em http://www.lbd.dcc.ufmg. br/colecoes /wei/2013/0033.pdf, Acesso em mar. 2015.

Libâneo, J. C. (2000) "As novas tecnologias da comunicação e informação, a escola e os professores", Adeus professor, adeus professora? Novas exigências educacionais e profissão docente, Cortês: São Paulo, p. 31.

Maloney, J., Peppler, K., Kafai, B. Y., Resnick, M. e Rusk, N. (2008) "Programming by choice: Urban Youth Learning Programming with Scratch", Anais do 39th SIGCSE'08, p. 367-371, Portland.

Maloney, J., Resnick, M., Rusk, N., Silverman, B. e Eastmond, E. (2010) "The scratch programming language and environment", ACM Transactions on Computing Education, vol. 10, n. 4.

Pereira, J. C. R. e Rapkiewicz, C. E. (2004) “O Processo de Ensino e Aprendizagem de Algoritmos e Programação: Uma Visão Crítica da Literatura”, Anais do III Workshop de Educação em Computação e Informática, Belo Horizonte.

Ramos, H. A. (2014) "Pensamento Computacional na Educação Básica: uma proposta de aplicação pedagógica para alunos do quinto ano do Ensino Fundamental do Distrito Federal", UnB: Brasília.

Silva, F. B., Romani, R. e Baranauskas, M. C. C. (2008) "SOO Brasileiro: Aprendizagem e Diversão no XO”, Revista Brasileira de Informática na Educação, p. 29-41. 